\title{
Hand Osteoarthritis Severity is Associated with Total Knee Joint Replacements Independently of BMI. The Ages-Reykjavik Study
}

\author{
Helgi Jonsson ${ }^{*}, 1$, Gudrun P. Helgadottir ${ }^{2}$, Thor Aspelund ${ }^{2,3}$, Gudny Eiriksdottir ${ }^{3}$, Sigurdur Sigurdsson ${ }^{3}$, \\ Kristin Siggeirsdottir ${ }^{3}$, Thorvaldur Ingvarsson ${ }^{4}$, Tamara B. Harris ${ }^{5}$, Lenore Launer ${ }^{5}$ and \\ Vilmundur Gudnason ${ }^{2,3}$
}

\author{
${ }^{1}$ Landspitalinn University Hospital, University of Iceland, Reykjavik, Iceland \\ ${ }^{2}$ University of Iceland, Reykjavik, Iceland \\ ${ }^{3}$ Icelandic Heart Association, Kopavogur, Iceland \\ ${ }^{4}$ Akureyri Central Hospital, Akureyri, Iceland. \\ ${ }^{5}$ National Institute on Aging, Bethesda, MD, USA
}

\begin{abstract}
Objective: To identify factors associated with having total knee replacement due to osteoarthritis in the AGESReykjavik Study, a large population based study of elderly Icelanders.

Methods: Information about total knee and hip joint replacements (TKR,THR) and hand OA (HOA) severity was available in 2195 males and 2975 females, mean age $76 \pm 6$ years. The prevalence of TKR was $223(4.3 \%)$ and THR 316 (6.1\%). We performed a backwards binary logistic regression analysis of possible OA associated variables including age, gender, abdominal circumference, BMI, hs-CRP, cholesterol, statin use, bone mineral density of the spine, education and smoking history as well as HOA severity and the presence of THR.

Results: Only three factors showed significant associations with TKR; BMI $\left(\mathrm{p}=3.5 \times 10^{-17}\right)$, HOA severity $\left(\mathrm{p}=2.9 \times 10^{-8}\right)$ and THR ( $\mathrm{p}=0.0002)$. The highest quintile of BMI was associated with a fivefold risk of TKR compared with the lowest $(8 \%$ vs $1.6 \%$ ), and severe HOA had a 2.4 fold risk compared with those with no HOA ( $8 \%$ vs 3.3\%). There was no statistical interaction between BMI and HOA. Thus, individuals with BMI $<23.5$ with no evidence of HOA had a prevalence of TKR of $1.1 \%$, while those with $\mathrm{BMI}>30.3$ and severe $\mathrm{HOA}$ had a prevalence of $13.4 \%$.

Conclusions: Hand and hip osteoarthritis in conjunction with BMI are strongly associated with the prevalence of TKR due to osteoarthritis. Together, BMI and HOA severity seem to contribute to the majority of the total TKR prevalence. While BMI has long been recognized as the major risk factor for TKR, the influence of osteoarthritis at other sites may have been underestimated.
\end{abstract}

Keywords: Epidemiology, knee osteoarthritis, knee joint replacement, Body Mass index (BMI), hand osteoarthritis, AGESReykjavík Study.

\section{BACKGROUND}

Body Mass Index (BMI) is the best known risk factor for the incidence and progression of knee osteoarthritis. High BMI seems to antedate the development of knee OA and a linear relationship has been demonstrated both in radiological studies and studies using total knee joint replacement (TKR) as a definition of severe OA [1-11]. The association is quite strong as demonstrated in a recent prospective study showing a RR of 8.1 for having TKR in individuals in the highest compared with the lowest quartile of BMI [10]. This relationship has a strong biomechanical component, although metabolic factors related to adipose tissue have also been suggested $[12,13]$.

*Address correspondence to this author at the Landspitalinn University Hospital, IS-108 Fossvogur, Reykjavik, Iceland; Tel: -354 543 6465; Fax: 354543 6568; E-mail: helgi@hi.is
Additional suggested risk factors include age, malalignment, laxity, trauma, smoking habits, physical activity, other measures of body weight and fat distribution, vitamin $\mathrm{D}$ deficiency, metabolic aberrations including the metabolic syndrome [13, 14], bone mineral density and osteoarthritis at other sites, but with the exception of BMI and significant trauma, reports are inconsistent $[1,8]$.

The presence of hand osteoarthritis (HOA) in conjunction with knee and hip OA, has been interpreted as a marker of "generalized osteoarthritis" but the definition of that particular condition remains elusive [15]. A number of studies have shown a relation between the presence of HOA and knee OA progression [2, 16-18].

The AGES-Reykjavik Study (Age, Gene/Environment Susceptibility - Reykjavik Study) is a population-based multidisciplinary study of aging in elderly individuals from the 40 year long Reykjavik Study [19]. Among the extensive 
baseline data gathered on more than 5700 elderly people is information about whether the participant had undergone total joint replacements of knees and hips (THR) and HOA (ascertained from digital photographs of the hands). The objective of the current study was the analysis of possible risk factors for having TKR due to osteoarthritis.

\section{METHODS}

The AGES-Reykjavik Study is a population based study of elderly individuals from the 40 year long Reykjavik Study. They were aged between 66 and 96 and randomly recruited between 2002 and 2006. Informed consent was obtained from all participants. Details of the investigations are described in the study's baseline article [19].

Knee and hip joint replacements (TKR,THR) were recorded on the basis of computerized tomography (CT) anterior scout scans. A total of 5250 individuals had both CT scans and high quality hand photographs for the assessment of HOA, and a total of 581 of those had total joint replacements (TJR). In order to identify those with total joint replacements (TJR) presumably due to osteoarthritis, subjects with evidence of inflammatory arthritis on the CT scans or photographs were excluded $(n=27)$. Furthermore, additional 53 photographs could not be scored for HOA due to contractures, deformities or thumb positioning. This left a study group of 5170 with a total of 559 subjects with TJR. Finally, this group was then compared with a verified fracture registry for this group based on medical records [20], excluding TJR's due to fractures. This left a study group of 5170 subjects (2195 males and 2975 females) 508 of whom had total joint replacements that were presumed to be caused by OA. The average age of the participants was 76 \pm 6 years.

The presence and severity of HOA was read from high quality digital photographs and scored as $0-3$ for the distal interphalangeal joints (DIP), the proximal interphalangeal joints (PIP) and the first carpometacarpal joint (CMC1) joints creating an aggregate score of $0-9$. This score was subsequently truncated to $0,1,2,3$ and $4+$ as previously described [21].

The other variables in this study have been described previously $[19,21]$ with the exception of "midlife BMI". This variable was recorded in the participants' previous visits in the Reykjavik Study between the ages 33-65. In the cases where more than one BMI measure was available, the one closest to 50 years of age was used. The mean age of this BMI measure was $51 \pm 6$ years. Midlife BMI information was available in 5083 of the 5170 participants.

\section{Statistics}

TKR is a binary variable and we performed a backwards binary logistic regression using previously suggested risk factors for TKR including age, gender, abdominal circumference, BMI, hs-CRP, cholesterol, statin use, bone mineral density of the spine, education and smoking history as well as HOA severity and the presence of THR. Age and gender stayed in the model. In order to analyze the association of THR to TKR, we considered THR as a binary variable for severe hip OA, and combined it with two other binary factors, severe hand OA, (defining those with a HOAscore $\geq 4$ as positive and the remainder negative) and high BMI (positive defined as the highest quintile of BMI, BMI $\geq 30.3$ ). The statistical analysis was done using the SPSS 16.0 statistical software package.

\section{RESULTS}

The total number of THR presumed to be due to OA was $316(6.1 \%), 206$ females and 110 males. 31 participants had both hip and knee replacements (Table 1) The total number of individuals with TKR was 223 (4.3\%), 142 females and 81 males.

The gender prevalence of TKR and THR is shown in Table 1.

Table 1. The Prevalence of Hip (THR) and Knee (TKR) Joint Replacements in the AGES-Reykjavik Study

\begin{tabular}{|c|c|c|c|}
\hline Gender & $\mathbf{n}$ & TKR (\%) & THR (\%) \\
\hline \hline Males & 2195 & $81(3.7)$ & $110(5.0)$ \\
\hline Females & 2975 & $142(4.8)$ & $206(6.9)$ \\
\hline All & 5170 & $223(4.3)$ & $316(6.1)$ \\
\hline
\end{tabular}

The backwards binary logistic regression of possible risk factors for TKR included age, gender, abdominal circumference, BMI, hs-CRP, cholesterol, statin use, bone mineral density of the spine, education and smoking history as well as HOA severity and the presence of THR. Age and gender stayed in the model. Only three of the variables investigated showed significant associations with TKR; BMI $\left(\mathrm{p}=3.5 \times 10^{-17}\right)$, HOA severity $\left(\mathrm{p}=2.9 \times 10^{-8}\right)$ and THR $(\mathrm{p}=0.0002)$. (Table 2$)$.

Table 2. Odds Ratios for TKR for Age, Gender and the Three Significantly Associated Variables

\begin{tabular}{|c|c|c|}
\hline Variable & OR (95\%CI) & $\mathbf{p}$ \\
\hline \hline Age (5 years) & $1.09(0.96-1.24)$ & $\mathrm{ns}$ \\
\hline Female sex & $1.09(0.82-1.45)$ & $\mathrm{ns}$ \\
\hline BMI (1 unit) & $1.13(1.10-1.16)$ & $3.5 \times 10^{-17}$ \\
\hline Hand OA score (1 unit) & $1.32(1.20-1.46)$ & $2.9 \times 10^{-8}$ \\
\hline THR (yes/no) & $2.20(1.46-3.32)$ & 0.0002 \\
\hline
\end{tabular}

The highest quintile of BMI (mean = 33.6) was associated with a fivefold increase $(8 \%$ vs $1.5 \%)$ in the prevalence of TKR compared with the lowest quintile of BMI (mean=21.4). Severe HOA had a 2.4 fold increase compared to those with no HOA ( $8 \%$ vs $3.3 \%)$. A statistically significant interaction between these two factors was not found $(\mathrm{P}=0.85)$. Both $\mathrm{BMI}$ and HOA are ordinal variables, and the relationship between quintiles of BMI and the HOA severity score in relation to the prevalence of TKR is shown in Table 3.

Thus, those with $\mathrm{BMI}<23.5$ and no evidence of $\mathrm{HOA}$ had a prevalence of TKR of $1.1 \%$, while individuals with BMI $>30.3$ and severe HOA had a prevalence of $13.4 \%$ showing a twelvefold prevalence of TKR in the latter group. 
Table 3. Cross-Tabulations of the Percent of Study Subjects with Total Knee Replacements in Relation to Quintiles of BMI and HOA Severity (Percent)

\begin{tabular}{|c|c|c|c|c|c|c|}
\hline \multirow{2}{*}{ BMI (Quintiles) } & \multicolumn{6}{|c|}{ HOAscore } \\
\hline & $0(n=1611)$ & $1(n=1170)$ & $2(n=1020)$ & $3(n=696)$ & $4+(n=673)$ & Total \% \\
\hline $1(<23.5)$ & 1.1 & 1.0 & 1.4 & 3.4 & 1.6 & 1.5 \\
\hline $2(23.5-25.6)$ & 1.9 & 1.3 & 4.3 & 3.2 & 6.2 & 3.0 \\
\hline $3(25.6-27.7)$ & 2.9 & 1.9 & 2.8 & 5.6 & 13.1 & 4.3 \\
\hline $4(27.7-30.3)$ & 3.2 & 3.1 & 5.8 & 4.3 & 11.4 & 4.7 \\
\hline $5(>30.3)$ & 6.8 & 5.9 & 7.0 & 13.2 & 13.4 & 8.0 \\
\hline Total\% & 3.3 & 2.8 & 4.2 & 5.7 & 8.0 & \\
\hline
\end{tabular}

For a better understanding of the relationship between BMI and TKR we did similar calculations using "midlife BMI" $(n=5087)$ instead of study BMI. Compared with Table 2 this resulted in an OR for TKR of 1.17 (1.14-1.21, $\mathrm{p}<1 \times 10^{-}$ $\left.{ }^{20}\right)$ per unit BMI. The relationship between midlife BMI, HOA severity and TKR is illustrated in Fig. (1).

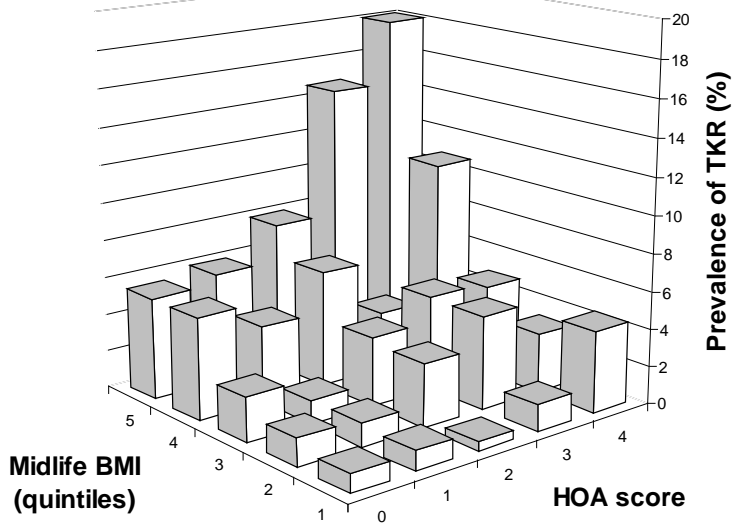

Fig. (1). A graphic illustration of the relationship between "midlife BMI" (quintiles) and hand osteoarthritis severity (HOA score 0-4) in relation to the prevalence of TKR in the study group $(n=5087)$.

Despite a higher prevalence of TKR and HOA in females, there was no discernible difference between men and women as shown in Fig. (2).

The presence of THR $(n=316)$ was also positively associated with the prevalence of TKR. Since this is a binary on-off variable, its relative contribution was examined with the two other variables associated with the highest prevalence of TKR, highest quintile of BMI $(n=1063)$ and severe HOA (HOAscore $\geq 4, n=673$ ). Fig. (3) illustrates this relationship, showing an increasing prevalence of TKR with the number of these three variables. Thus, individuals with none of the three factors positive had a TKR prevalence of $2.5 \%$, those with one positive $6.9 \%$ and those with two or more had a prevalence of TKR of $13.3 \%$.

\section{DISCUSSION}

In this cross-sectional, population-based study of 5170 elderly individuals there were strong associations between hand and hip osteoarthritis and the prevalence of TKR due to osteoarthritis. These associations appeared to be statistically independent of the effects of BMI. In the case of HOA, there was a linear association between HOA severity and TKR, and severe HOA and THR had similar odds ratios of 2.2-2.4 for having TKR.

a) Females $(\mathrm{n}=2975)$

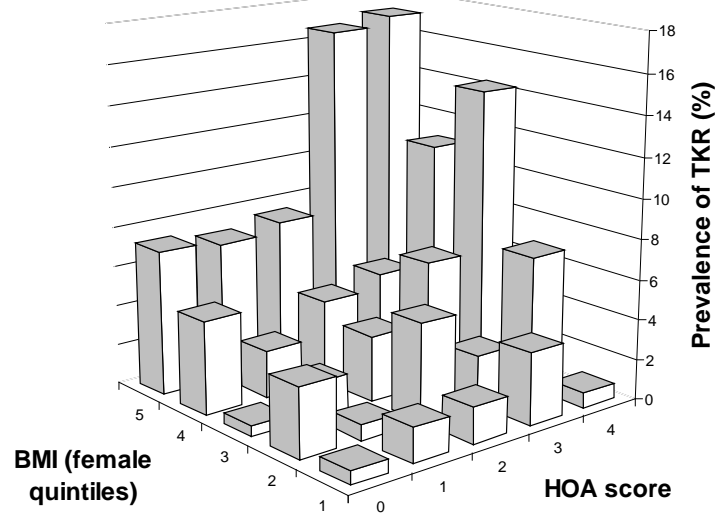

b) Males $(\mathrm{n}=2175)$

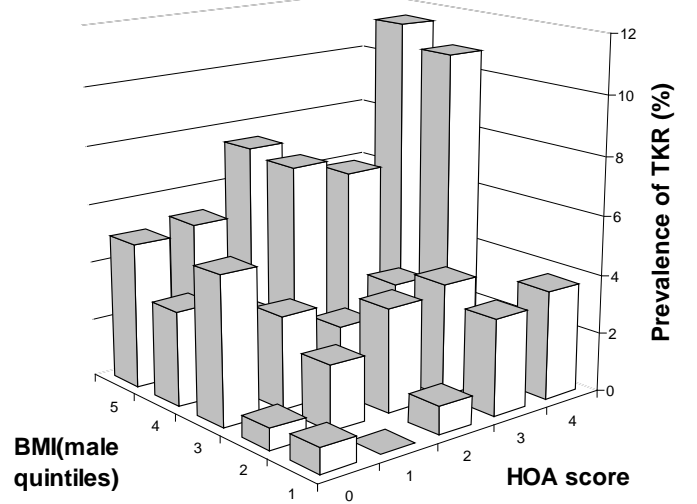

Fig. (2). Diagrams illustrating the similarity of the relationship in both genders despite higher prevalence of hand OA and TKR in females. a) Females ( $\mathrm{n}=2975)$, b) Males $(\mathrm{n}=2175)$.

The most striking new information in the current study is the strength and the linearity of the statistical associations between the two factors HOA severity and BMI on one side and the prevalence of TKR on the other. This relationship was similar in both genders. The statistical absence of associations with other possible risk factors apart from THR is also notable. It is tempting to speculate whether HOA and THR can be of help in identifying patients at risk for needing TKR. Combining the odds ratios associated with HOA severity and those associated with high BMI yielded very 
high odds ratios. If the data for those with no HOA and lowest quintile BMI are extrapolated to the whole study group, the calculated expected total number of TKR would be 62 instead of the 223 observed $(27.8 \%)$ indicating that these two major factors, BMI and HOA severity, may contribute to over $70 \%$ of total knee joint replacements in this age group. This was also supported by the "area under curve" (C-statistics) for the regression analysis (data not shown).

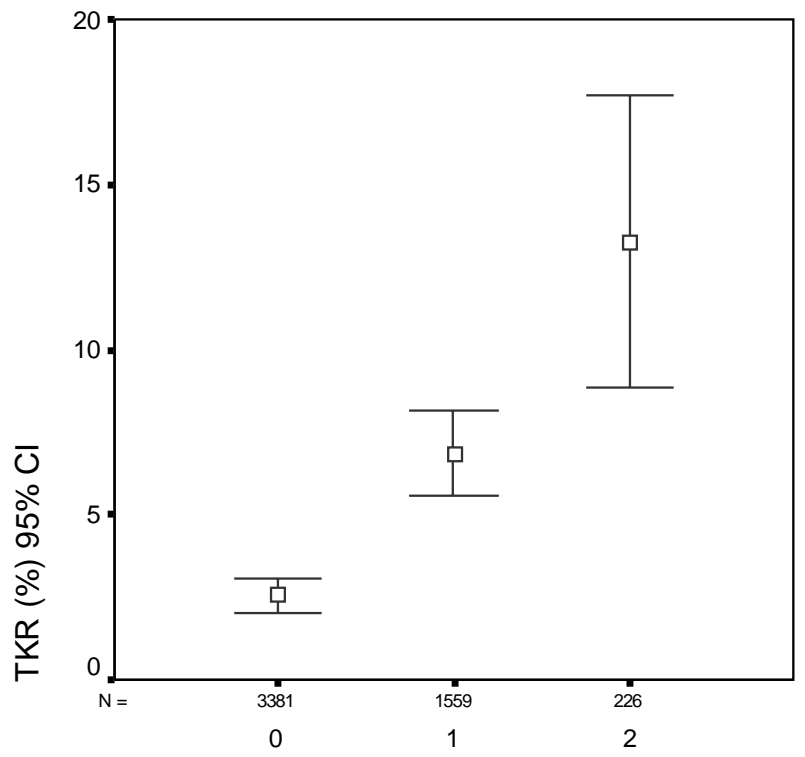

Number of Risk Factors

Fig. (3). The prevalence of TKR in relation to the presence or absence of the three major associated variables, high BMI, severe HOA and THR. Thus, individuals with none of the three factors had a TKR prevalence of $2.5 \%$, those with one factor positive $6.9 \%$ and those with two or more had a TKR prevalence of $13.3 \%$.

The role of BMI as the strongest risk factor for having TKR was confirmed in this study. Both current BMI, and "midlife BMI" showed a strong linear association with the prevalence of TKR. This indicates that high BMI antedates the need for TKR and the current findings are very much in analogy with recent prospective studies regarding risk estimates [10, 11]. In the absence of preventive drug treatment for $\mathrm{OA}$, weight reduction is one of the cornerstones of current knee OA management [22, 23]. Our findings might be used as an argument for recommending weight supervision in HOA patients even in the absence of knee OA. THR is less likely to be useful in predicting TKR because of the low numbers and the fact that THR and TKR operations are relatively late events in OA.

TKR is widely used as a separate phenotype of severe knee OA, particularly in genetic studies [24]. The current findings seem to cast some doubt on this definition, with the majority of TKR associated with BMI and osteoarthritis at other sites, and certainly these major factors need to be corrected for in genetic studies. The hard to define concept of generalized osteoarthritis might be the most appropriate target for genetic studies, but although this study does suggest a linear trend between the need for TKR and severity of hand OA, it does not really help in determining a cut-off point for generalized osteoarthritis. Other markers that may be useful for that definition include better OA imaging, genetic data, biomarkers and relationships with other conditions including finger length ratios [25] and concomitant disease conditions. Our group has recently described a linear association between HOA severity and atherosclerosis in the same population but only in females suggesting that systemic factors only active in females may apply as well [21].

The AGES-Reykjavik study, with its large number of elderly subjects is eminently suitable for analysis of this kind of relationships i.e. organ damage acquired during lifetime. The governmental health care system is socialized, ensuring universal access to medical care and the study itself is population-based, indicating that the current findings are probably representative of the Icelandic population. A number of other factors may have contributed to the distinct findings of the current study. They include a definite OA endpoint in TKR and a relatively moderate BMI compared with some other studies where BMI may be a more dominant cause of knee OA. Finally, it is possible that the use of photographs for the diagnosis of HOA could have identified slightly different cases compared with other studies [26]. While the strength of the study is the large size and uniform age, some caveats have to be considered. The main drawback of the study is its cross-sectional nature, limiting assumptions about the the predictive value of osteoarthritis at other sites. We also did not have reliable data on some risk factors, such as trauma or physical activity. The number of subjects in the group with both THR and TKR is low and even a small number of misdiagnoses such as seronegative arthritis or dysplastic conditions could seriously affect the odds ratios for this group. Also, the prevalence of TKR in this study does not represent true lifetime risk, as the incidence of new TKR operations is probably still high in the study age group [27]. Further studies of the longitudinal relationship are needed. We hope to be able to provide more information as the five year follow-up of the AGESReykjavik Study will be completed soon.

\section{CONCLUSION}

Hand and hip osteoarthritis in conjunction with BMI are strongly associated with the prevalence of TKR due to osteoarthritis. Although BMI has long been recognized as the major risk factor for TKR, the influence of osteoarthritis at other sites may have been underestimated, particularly that of hand osteoarthritis severity which has a linear relationship with the prevalence of TKR.

\section{ETHICAL APPROVAL AND INFORMED CONSENT}

The study is approved by the Icelandic National Bioethics Committee, (VSN: 00-063) and the Data Protection Authority. All participants signed an informed consent declaration.

\section{ACKNOWLEDGEMENTS}

The Age, Gene/Environment Susceptibility Reykjavik Study (AGES-Reykjavik) has been funded by NIH contract N01-AG-12100, the NIA Intramural Research Program, Hjartavernd (the Icelandic Heart Association), and the Althingi (the Icelandic Parliament), the Icelandic Osteoarthritis Fund and the University of Iceland Research 
Fund. The researchers are indebted to the participants for their willingness to participate in the study.

\section{CONFLICT OF INTEREST}

None

\section{AUTHOR CONTRIBUTIONS}

All authors have made some contributions to the text of the manuscript.

Helgi Jonsson: Main author.

Gudrun P. Helgadottir: Data acquisition, Manuscript preparation.

Thor Aspelund. Statistical analysis, Critical review of manuscript.

Gudny Eiriksdottir. Project Management, Critical review of manuscript.

Sigurdur Sigurdsson. Data acquisition, Critical review of manuscript.

Kristin Siggeirsdottir. Data acquisition, Critical review of manuscript.

Thorvaldur Ingvarsson, Data acquisition, Critical review of manuscript.

Tamara B. Harris. Project Management, Critical review of manuscript.

Lenore Launer. Project Management, Critical review of manuscript.

Vilmundur Gudnason. Project Management, Critical review of manuscript.

Hand OA, BMI and Total Knee Replacements

\section{ABBREVIATIONS}

$\begin{array}{ll}\text { OA } & =\text { Osteoarthritis } \\ \text { BMI } & =\text { Body Mass Index } \\ \text { TKR } & =\text { Total knee replacement } \\ \text { THR } & =\text { Total hip replacement } \\ \text { HOA } & =\text { Hand osteoarthritis } \\ \text { hs-CRP } & =\text { High sensitivity C- Reactive Protein } \\ \text { RR } & =\text { Relative Risk } \\ \text { OR } & =\text { Odds Ratio } \\ \text { DIP } & =\text { Distal interphalangeal joints } \\ \text { PIP } & =\text { Proximal interphalangeal joints } \\ \text { CMC1 } & =\text { First carpometacarpal joints } \\ \text { HOAscore } & =\text { A cumulative score for hand OA severity }\end{array}$

\section{REFERENCES}

[1] Felson DT, Lawrence RC, Dieppe PA, et al. Osteoarthritis: new insights. Part 1: The disease and its risk factors. Ann Intern Med 2000; 133: 635-46.

[2] Schouten JS, van den Ouweland FA, Valkenburg HA. A 12 year follow up study in the general population on prognostic factors of cartilage loss in osteoarthritis of the knee. Ann Rheumatol Dis 1992; 51: 932-7.

[3] Abbate LM, Stevens J, Schwartz TA, Renner JB, Helmick CG, Jordan JM. Anthropometric measures, body composition, body fat distribution, and knee osteoarthritis in women Obesity (Silver Spring) 2006; 14: 1274-81.

[4] Sowers MF, Yosef M, Jamadar D, Jacobson J, Karvonen-Gutierrez C, Jaffe M. BMI $v s$ body composition and radiographically defined osteoarthritis of the knee in women: a 4-year follow-up study. Osteoarthritis Cartilage 2008; 16: 367-72.

[5] Niu J, Zhang YQ, Torner J, et al. Is obesity a risk factor for progressive radiographic knee osteoarthritis? Arthritis Rheumatol 2009; 61: 329-35.

[6] Cicuttini FM, Spector T, Baker J. Risk factors for osteoarthritis in the tibiofemoral and patellofemoral joints of the knee. J Rheumatol 1997; 24: 1164-7.

[7] Grotle M, Hagen KB, Natvig B, Dahl FA, Kvien TK. Obesity and osteoarthritis in knee, hip and/or hand: an epidemiological study in the general population with 10 years follow-up. BMC Musculoskelet Disord 2008; 9: 132.

[8] Stürmer T, Günther KP, Brenner H. Obesity, overweight and patterns of osteoarthritis: the Ulm Osteoarthritis Study. J Clin Epidemiol 2000; 53: 307-13

[9] Franklin J, Ingvarsson T, Englund M, Lohmander LS. Sex differences in the association between body mass index and total hip or knee joint replacement resulting from osteoarthritis. Ann Rheumatol Dis 2009; 68: 536-40.

[10] Lohmander LS, Gerhardsson M, Rollof J, Nilsson PM, Engstrom G. Incidence of severe knee and hip osteoarthritis in relation to different measures of body mass. A population-based prospective cohort study. Ann Rheumatol Dis 2009; 68: 490-6.

[11] Wang Y, Simpson JA, Wluka AE, et al. Relationship between body adiposity measures and risk of primary knee and hip replacement for osteoarthritis: a prospective cohort study. Arthritis Res Ther 2009; 11 (3): R86.

[12] Pottie P, Presle N, Terlain B, Netter P, Mainard D, Berenbaum F Obesity and osteoarthritis: more complex than predicted! Ann Rheumatol Dis 2006; 65: 1403-5.

[13] Masuko K, Murata M, Suematsu N, et al. A metabolic aspect of osteoarthritis: lipid as a possible contributor to the pathogenesis of cartilage degradation. Clin Exp Rheumatol 2009; 27: 347-53.

[14] Gandhi R, Razak F, Tso P, Davey JR, Mahomed NN. Asian Ethnicity and the Prevalence of Metabolic Syndrome in the Osteoarthritic Total Knee Arthroplasty Population. J Arthroplasty 2010; 25 (3): 416-9.

[15] Vignon E. Hand osteoarthritis and generalized osteoarthritis: A need for clarification. Osteoarthritis Cartilage 1995; 8 (Supplement 1): S22-S24.

[16] Doherty M, Watt I, Dieppe P. Influence of primary generalised osteoarthritis on development of secondary osteoarthritis. Lancet 1983; 2: 8-11.

[17] Dahaghin S, Bierma-Zeinstra SM, Reijman M, Pols HA, Hazes JM, Koes BW. Does hand osteoarthritis predict future hip or knee osteoarthritis? Arthritis Rheumatol 2005; 52: 3520-7.

[18] Englund M, Paradowski PT, Lohmander LS. Association of radiographic hand osteoarthritis with radiographic knee osteoarthritis after meniscectomy. Arthritis Rheumatol 2004; 50: 469-75.

[19] Harris TB, Launer LJ, Eiriksdottir G, et al. Age, Gene/Environment Susceptibility-Reykjavik study: Multidiscipliary Applied Phenomics. Am J Epidemiol 2007.

[20] Siggeirsdottir K, Aspelund T, Sigurdsson G, et al. Inaccuracy in self-report of fractures may underestimate association with health outcomes when compared with medical record based fracture registry. Eur J Epidemiol 2007; 22: 631-9.

[21] Jonsson H, Helgadottir GP, Aspelund T, et al. Hand osteoarthritis in older women is associated with carotid and coronary atherosclerosis: The AGES - Reykjavik study. Ann Rheumatol Dis 2009; 68: 1696-700.

[22] Messier SP, Loeser RF, Miller GD, et al. Exercise and dietary weight loss in overweight and obese older adults with knee osteoarthritis: the Arthritis, Diet, and Activity Promotion Trial. Arthritis Rheumatol 2004; 50: 1501-10.

[23] Hooper MM, Stellato TA, Hallowell PT, Seitz BA, Moskowitz RW. Musculoskeletal findings in obese subjects before and after weight loss following bariatric surgery. Int J Obes (Lond) 2007; 31: 114-20.

[24] Evangelou E, Chapman K, Meulenbelt I, et al. Large-scale analysis of association between GDF5 and FRZB variants and osteoarthritis of the hip, knee, and hand. Arthritis Rheumatol 2009; 60: 1710-21. 
[25] Zhang W, Robertson J, Doherty S, et al. Index to ring finger length raoti and the risk for osteoarthritis. Arthritis Rheumatol 2008; 58: $137-44$.

[26] Helgadottir GP, Eliasson GJ, Jonsson A, et al. Comparison of photographs, clinical examination and radiographs for the assessment of hand osteoarthritis. Osteoarthritis Cart 2007; 15 (Supplement C): C186.

[27] The Swedish Knee Arthroplasty Register 2008. http://knee.nko.se/ english/online/

Received: July 8, 2010

(C) Jonsson et al.; Licensee Bentham Open.

This is an open access article licensed under the terms of the Creative Commons Attribution Non-Commercial License (http://creativecommons.org/licenses/by-nc/

3.0/) which permits unrestricted, non-commercial use, distribution and reproduction in any medium, provided the work is properly cited. 